\title{
New treatment options for nonmetastatic osteosarcoma: focus on mifamurtide in adolescents
}

This article was published in the following Dove Press journal:

Clinical Oncology in Adolescents and Young Adults

5 September 2012

Number of times this article has been viewed

\author{
Winston W Huh \\ Daniela Egas-Bejar \\ Peter MAnderson \\ Division of Pediatrics, The Children's \\ Cancer Hospital of the University of \\ Texas MD Anderson Cancer Center, \\ Houston, TX, USA
}

\begin{abstract}
New treatments are needed to improve the clinical outcome for patients with osteosarcoma. Liposomal muramyl tripeptide phosphatidylethanolamine (mifamurtide) is a synthetic peptidoglycan component packaged in multilamellar liposomes. Mifamurtide has been demonstrated to induce recruitment and activation of macrophages and monocytes of the host innate immune system, which leads to antitumoricidal activity. Early clinical trials have demonstrated the safety and tolerability of mifamurtide combined with chemotherapy, and one major study has demonstrated an overall survival benefit in patients with newly diagnosed nonmetastatic osteosarcoma. This review summarizes the mechanism of action, clinical results, and the optimal biologic dose, and raises potential questions for future development of mifamurtide.
\end{abstract}

Keywords: osteosarcoma, childhood cancer, adolescent, sarcoma, immunotherapy, bone

\section{Introduction}

Osteosarcoma is the most common malignant bone tumor occurring in children and adolescents. The peak age of occurrence is during the second decade of life, and in the US the incidence is 4.4 cases per million individuals younger than 25 years. ${ }^{1}$ The long bones of the extremities, especially at the metaphyses, are the most common sites involved. The treatment of osteosarcoma is multidisciplinary in nature and involves careful coordination of complete surgical removal of the primary tumor combined with 6-9 months of systemic chemotherapy given before surgery (neoadjuvant) and also after surgery (adjuvant). Currently, there are four chemotherapeutic agents that are considered active standard agents for the treatment of osteosarcoma, ie, doxorubicin, cisplatin, high-dose methotrexate with leucovorin rescue, and ifosfamide. Recent studies evaluating treatment regimens from the 1980s and 1990s using the standard cytotoxic chemotherapeutic agents have shown that the overall survival rates for patients with nonmetastatic osteosarcoma have remained at approximately $60 \%-70 \% .^{2-5}$ Thus, the consensus has been that a plateau of efficacy has been reached with traditional cytotoxic chemotherapy regimens and that new agents or therapies are required to improve the survival outcome for patients with osteosarcoma.

Ever since William B Coley's initial description of tumor response in three patients with bone sarcomas following injection of streptococcal organisms in 1891, there has been significant interest in the role of enhancing the innate immune system for solid tumor therapy. ${ }^{6}$ A recent study found that among 412 patients with osteosarcoma, those who experienced a postoperative infection within one year of their limb salvage 
surgery had a significantly improved 10-year overall survival outcome compared with those patients without a history of infection ( $84 \%$ versus $62 \%$ )..$^{7}$ This finding is intriguing considering that infections of the extremities had an effect against distant lung metastases. However, another recent clinical study in 31 patients with shorter follow-up did not find a relationship between postoperative infection and improved clinical outcome for patients with osteosarcoma. ${ }^{8}$ Other data supporting the potential involvement of the host immune system against tumor cells include the observation of early lymphocyte recovery correlating with improved clinical outcome in patients with bone tumors. ${ }^{9,10}$ Previous studies have investigated the potential of immunostimulatory agents, such as interferon and granulocyte-macrophage colony stimulating factor, for patients with osteosarcoma, but the results of these studies have been conflicting at best. ${ }^{1-14}$ However, it is likely that a variety of clinical factors, such as extent of disease burden, are involved in determining the potential efficacy of enhanced immunity for tumor therapy. Much investigation is still needed regarding the potential benefit of agents that enhance innate immunity in patients with non-metastatic versus metastatic disease and how to integrate immune modulators with traditional therapies for osteosarcoma.

Muramyl tripeptide phosphatidylethanolamine (MTP-PE) is a synthetic derivative of muramyl dipeptide (MDP), which is a peptidoglycan component found in bacterial cell walls. ${ }^{15,16}$ Mifamurtide is a formulation of MTP-PE encapsulated into multilamellar liposomes (L-MTP-PE) and functions as a much more potent activator of macrophages and monocytes than MDP, and L-MTP-PE is also less toxic than MDP. ${ }^{17-19}$ Much work has already been done to demonstrate that mifamurtide potentiates the tumoricidal ability of macrophages and monocytes. Given that the lungs harbor a significant population of macrophages and are the most common site of metastatic involvement and recurrence of osteosarcoma, there is a rationale for developing mifamurtide as a new therapeutic agent for osteosarcoma. ${ }^{3,20,21}$ The purpose of this review is to provide background on the clinical development of mifamurtide and also to discuss the potential use and safety of mifamurtide in adolescents and young adults with osteosarcoma.

\section{Chemistry, pharmacokinetics, and pharmacodynamics}

Mifamurtide is produced by combining the active MTP-PE agent with synthetic phospholipids, ie, dioleoyl phosphatidylserine and palmitoyl oleoyl phosphatidylcholine, and then lyophilized to form concentric multilamellar liposomes measuring 2-3 $\mu \mathrm{m}$ in diameter. ${ }^{22}$ The phosphatidylserine component binds to corresponding receptors on macrophages, and the liposomes are ingested via phagocytosis. ${ }^{23}$ Thus, the distribution of mifamurtide is biased towards organs with heavy concentrations of macrophages and monocytes, such as the liver, lungs, and spleen. This preferential distribution has been demonstrated in patients given mifamurtide labeled with ${ }^{99} \mathrm{Tc} .{ }^{24}$ The drug is rapidly cleared from the serum, with one study of adult patients demonstrating free MTP-PE serum concentrations at less than $1 \%$ of the administered dose when measured 30 minutes after infusion. ${ }^{25}$ While there have not been any studies specifically evaluating the pharmacokinetics of mifamurtide in children and adolescents, a study by Murray et al included older adolescents at least 18 years of age and young adults, and demonstrated rapid distribution of ${ }^{99} \mathrm{Tc}$-labeled mifamurtide to the reticuloendothelial system within 6 hours. ${ }^{24}$

As each layer of the liposomes degrades, MTP-PE is released within the cell. The main intracellular mechanism of action is felt to be mediated by binding of MTP-PE to the nucleotide-binding oligomerization domain (Nod) 2 receptor, which is highly expressed in antigen-presenting cells. ${ }^{26,27}$ Stimulation of Nod2 has been shown to result in activation of the nuclear factor- $\kappa \mathrm{B}$ signaling pathway and also increased secretion of interleukin (IL)-1 $\beta$, leading to activation of the mitogen-activated protein kinase pathway. ${ }^{28,29}$

Activation of macrophages and monocytes results in the secretion of cytokines and other proinflammatory molecules, including IL-1, IL-6, IL-8, tumor necrosis factor alpha, nitric oxide, and prostaglandins $\mathrm{D}_{2}$ and $\mathrm{E}_{2} \cdot{ }^{30-34}$ Increased expression of intracellular adhesion molecules, such as lymphocyte function-associated antigen-1 and intracellular adhesion molecule-1, have also been demonstrated on MTP-PE exposure to human monocytes. ${ }^{35}$ Upregulation of these molecules likely leads to activation of contactmediated tumoricidal function by innate immune cells. The immune-mediated tumor killing mechanism of mifamurtide is supported by in vivo studies demonstrating that following mifamurtide treatment, osteosarcoma tumors demonstrate a pattern of necrosis similar to that of tuberculous granulomas. ${ }^{36}$

A number of in vitro and in vivo studies have not demonstrated any significant adverse interactions between mifamurtide and a variety of chemotherapy agents. ${ }^{37-41}$ Also, addition of chemotherapy agents, such as doxorubicin and ifosfamide, does not negatively affect the ability of MTP-PE to activate macrophages. ${ }^{37,39}$ However, high doses of 
ibuprofen have been found to inhibit the immunomodulatory effects of mifamurtide. ${ }^{42} \mathrm{~A}$ more comprehensive overview of the immunomodulatory and tumoricidal effects and the pharmacokinetics of mifamurtide has been detailed in several excellent reviews. ${ }^{22,43,44}$

\section{Clinical review}

Early clinical trials in adult and pediatric patients in a variety of malignancies have demonstrated that mifamurtide is relatively well tolerated with little evidence of acute or long-term organ toxicity. Fever, chills, headache, and fatigue have been the most commonly reported toxicities, occurring shortly following infusion, and these symptoms correspond to the immune-mediated proinflammatory response generated by mifamurtide (Table 1). ${ }^{24,45-49}$ Elevations in IL-6 have correlated with the onset of fever. ${ }^{47}$ Also, some of the infusion-related effects appear to be dose-related. Significant malaise and hypotension were more commonly observed in patients receiving doses greater than $2 \mathrm{mg} / \mathrm{m}^{2} \cdot{ }^{24,49}$ Most of these symptoms are readily addressed by administration of anti-inflammatory medications, such as acetaminophen or ibuprofen given at standard doses. Other toxicities that were reported included dyspnea, hypertension, anorexia, vertigo, diarrhea, leg cramps, and joint pain. ${ }^{24,46}$

The most significant clinical trial demonstrating the benefit of mifamurtide for osteosarcoma was the randomized, prospective Intergroup 0133 study for patients with newly diagnosed osteosarcoma. ${ }^{48,50}$ The trial had two main study questions which were posed using a $2 \times 2$ factorial design. The first objective was to determine if addition of ifosfamide to the three-drug combination of doxorubicin, cisplatin, and highdose methotrexate would improve the outcome. The second

Table I Most commonly reported acute infusion-related toxicities for mifamurtide

\begin{tabular}{|c|c|c|c|}
\hline References & Study population & L-MTP-PE dosing & $\begin{array}{l}\text { Summary of infusion-related } \\
\text { toxicities }\end{array}$ \\
\hline Murray et $\mathrm{al}^{24}$ & $\begin{array}{l}\text { Phase I study of } 28 \text { adult patients } \\
\text { with metastatic cancer. Most } \\
\text { common diagnoses were colorectal } \\
(n=10) \text { and renal cell carcinoma } \\
(n=5) \text {. }\end{array}$ & $\begin{array}{l}\text { Starting dose of } 0.05 \mathrm{mg} / \mathrm{m}^{2} \\
\text { biweekly for } 3 \text { weeks and } \\
\text { then escalated up to } 12 \mathrm{mg} / \mathrm{m}^{2} \text {. }\end{array}$ & $\begin{array}{l}\text { The MTD was } 6 \mathrm{mg} / \mathrm{m}^{2} \text {. } \\
\text { At the MTD, the most common } \\
\text { toxicities encountered were chills, } \\
\text { fever, malaise, nausea/vomiting, } \\
\text { anorexia, headache, myalgia, and cough. } \\
\text { Two patients experienced } \\
\text { grade } 3-4 \text { toxicities at a dose of } \\
3 \mathrm{mg} / \mathrm{m}^{2} \text {, ie, one patient with fever, } \\
\text { hypotension, and dyspnea and } \\
\text { another patient with dyspnea. }\end{array}$ \\
\hline Creaven et $\mathrm{al}^{46}$ & $\begin{array}{l}\text { Phase I study of } 37 \text { adult patients. } \\
\text { Most common diagnoses were renal } \\
\text { cell carcinoma }(n=13) \text { and } \\
\text { melanoma }(n=6)\end{array}$ & $\begin{array}{l}\text { Dosing of } 0.01-6 \mathrm{mg} / \mathrm{m}^{2} \\
\text { biweekly for } 4 \text { weeks. }\end{array}$ & $\begin{array}{l}\text { The MTD was considered to be } \\
2-4 \mathrm{mg} / \mathrm{m}^{2} \text {. At the MTD, the most } \\
\text { common toxicities encountered } \\
\text { were rigors, tachycardia, tachypnea, } \\
\text { nausea/vomiting, cyanosis, headache, } \\
\text { hypotension, and fatigue. }\end{array}$ \\
\hline Urba et $\mathrm{al}^{49}$ & $\begin{array}{l}\text { Phase I study of } 27 \text { adult patients. } \\
\text { Most common diagnoses were } \\
\text { colorectal }(n=15) \text {, renal cell } \\
\text { carcinoma }(n=4) \text {, and } \\
\text { melanoma }(n=4) \text {. }\end{array}$ & $\begin{array}{l}\text { Dosing of } 0.1-2.7 \mathrm{mg} / \mathrm{m}^{2} \\
\text { weekly for } 8 \text { weeks. }\end{array}$ & $\begin{array}{l}\text { The MTD was not reached. } \\
\text { Most common toxicities reported } \\
\text { were fever and rigors. } \\
\text { Hypotension noted in two patients } \\
\text { treated at } 2.7 \mathrm{mg} / \mathrm{m}^{2} \text { dose. }\end{array}$ \\
\hline Kleinerman et $\mathrm{al}^{47}$ & $\begin{array}{l}\text { Phase II study of } 16 \text { patients } \\
\text { with osteosarcoma. }\end{array}$ & $\begin{array}{l}2 \mathrm{mg} / \mathrm{m}^{2} \text { biweekly for } 12 \text { weeks } \\
\text { and then weekly for } 12 \text { weeks. }\end{array}$ & $\begin{array}{l}\text { Fevers, rigors, and headache were } \\
\text { the most common toxicities. }\end{array}$ \\
\hline Kleinerman et $\mathrm{al}^{45}$ & $\begin{array}{l}\text { Phase Ilb study of } 9 \text { patients } \\
\text { with osteosarcoma. }\end{array}$ & $\begin{array}{l}\text { Combined with ifosfamide. } \\
\text { Dose of } 2 \mathrm{mg} / \mathrm{m}^{2} \text { biweekly for } \\
12 \text { weeks and then weekly for } \\
12 \text { weeks. }\end{array}$ & $\begin{array}{l}\text { Fever, chills, myalgias, fatigue, and } \\
\text { headache were most common } \\
\text { toxicities reported. Two patients } \\
\text { with pre-existing history of asthma } \\
\text { experienced wheezing in first } 2 \\
\text { weeks of therapy but resolved with } \\
\text { bronchodilators. }\end{array}$ \\
\hline Meyers et $\mathrm{al}^{48}$ & $\begin{array}{l}\text { Phase III study of } 662 \text { patients } \\
\text { with nonmetastatic osteosarcoma. }\end{array}$ & $\begin{array}{l}\text { Combined with MAP } \pm \text { ifosfamide. } \\
\text { Starting at week } 12 \text {, dosing was } \\
2 \mathrm{mg} / \mathrm{m}^{2} \text { biweekly for } 12 \text { weeks } \\
\text { and then weekly for } 24 \text { weeks. }\end{array}$ & $\begin{array}{l}\text { Fever and chills were most common } \\
\text { toxicities reported. }\end{array}$ \\
\hline
\end{tabular}

Abbreviations: L-MTP-PE, liposomal muramyl tripeptide phosphatidyl ethanolamine; MTD, maximum tolerated dose; MAP, doxorubicin, cisplatin, and methotrexate. 
objective was to determine if addition of mifamurtide with chemotherapy would be of additional benefit. Those patients assigned to receive the drug started receiving mifamurtide after completing neoadjuvant chemotherapy and having their primary tumor surgically resected. The dosing schedule was twice weekly for 12 weeks then weekly for 24 weeks; thus, the total number of doses administered was 48 doses over a 36-week period.

When reviewing the results for patients with nonmetastatic disease, the study determined that addition of ifosfamide did not appear to confer an outcome benefit. However, addition of mifamurtide resulted in a significant improvement in 6 -year overall survival $(78 \%$ with mifamurtide versus $70 \%$ without mifamurtide; $P=0.03) .{ }^{50}$ Interestingly, when also reviewing the outcome in patients with metastatic osteosarcoma, there did appear to be a possible trend toward improvement in 5-year overall survival for those patients who received mifamurtide $(53 \%)$ versus those who did not receive mifamurtide $(40 \% ; P=0.19)$, but this did not achieve statistical significance, likely due to the small cohort size. ${ }^{51}$ A compassionate access trial for patients with high-risk osteosarcoma has recently completed enrollment in the US ( $n=200$ patients), and the data from this trial are currently being analyzed. At this time, mifamurtide is approved for use as treatment for nonmetastatic osteosarcoma in Europe, while in the US mifamurtide has not yet received approval from the Food and Drug Administration.

\section{Future directions}

While mifamurtide has demonstrated some promise in the treatment of osteosarcoma, there remain questions regarding which clinical scenarios will benefit the most from mifamurtide therapy. Although preclinical work has indicated that the best results with enhanced immune therapy are seen in the setting of low disease burden, the best means to achieve minimal disease status rapidly remains to be determined. While the combination of mifamurtide with cytotoxic chemotherapy has been studied, combination of mifamurtide with other therapeutic agents, such as radiopharmaceuticals, has not been evaluated and provides an avenue for additional studies. Samarium-153 ethylenediamine-tetramethylene phosphonic acid ( ${ }^{153} \mathrm{Sm}$-EDTMP) is a bone-seeking radiopharmaceutical agent that has been used in patients with osteosarcoma and bone metastases. ${ }^{52,53}$ Thus, one potential scenario for future study may be the addition of mifamurtide following treatment with ${ }^{153} \mathrm{Sm}$-EDTMP or another bone-seeking radiopharmaceutical, such as radium-223.

Another area for further study is the combination of mifamurtide with other immunomodulatory agents, such as

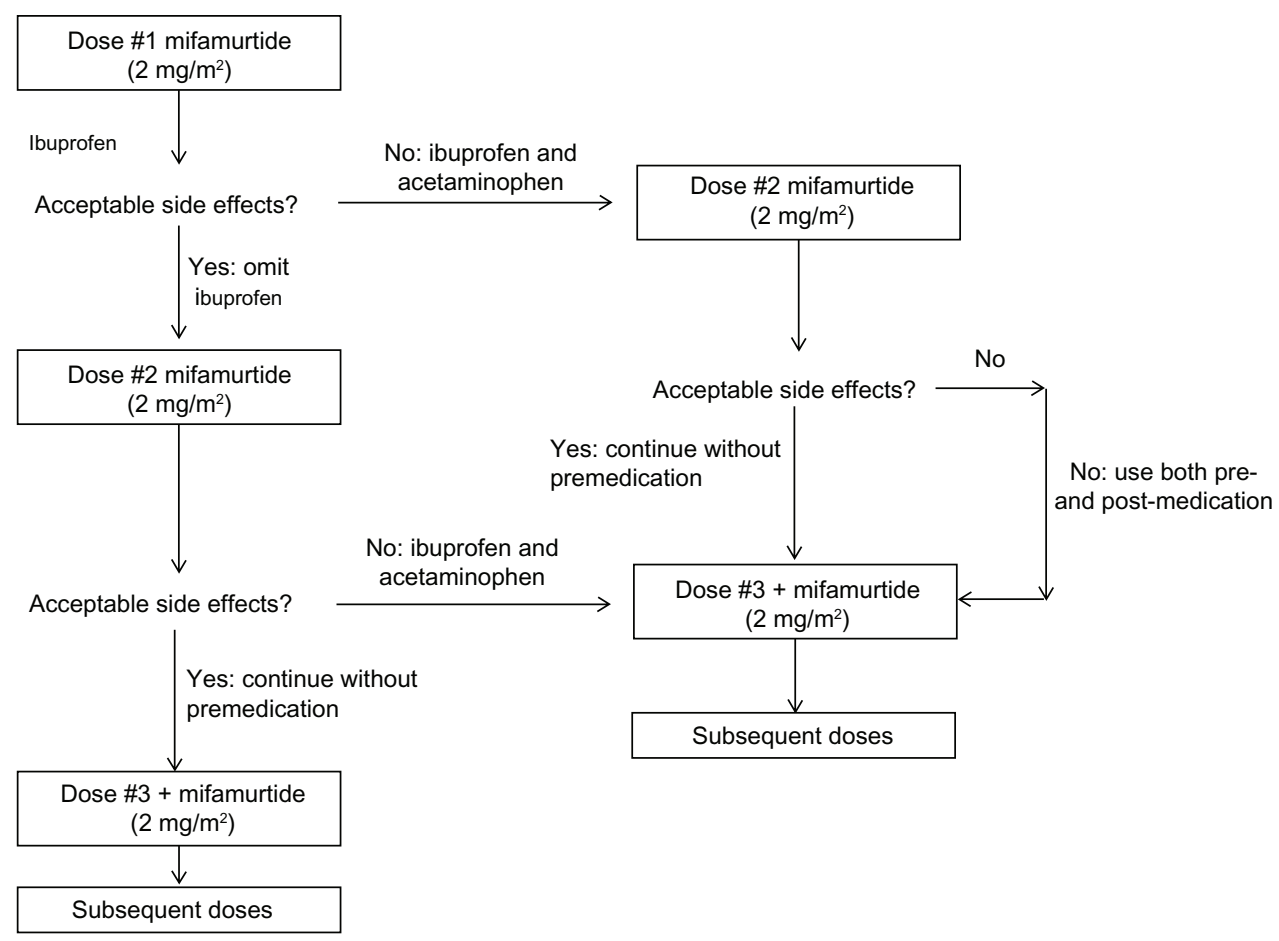

Figure I Algorithm for treating infusion-related side effects of mifamurtide.

Notes: Although premedication is often needed to reduce side effects after the first dose, most patients will require less premedication for subsequent doses. Occasional patients will require both before and after medication to reduce infusion-related symptoms. 
inhaled granulocyte-macrophage colony stimulating factor. ${ }^{11}$ A recent Phase I clinical trial by the Children's Oncology Group evaluating inhaled granulocyte-macrophage colony stimulating factor demonstrated its safety and tolerability in pediatric patients but was considered clinically ineffective. ${ }^{11}$ However, the combination of mifamurtide with inhaled immunomodulators or harvested immune cells, such as natural killer cells or chimeric antigen receptor $\mathrm{T}$ cells, may provide synergy in the recruitment and activation of macrophages and monocytes. While there may be concern that the combination of such agents could result in an increased likelihood of side effects, such as fever, chills, and myalgias, prompt treatment with nonsteroidal antiinflammatory medications would likely ameliorate the fever and myalgias, while meperedine would address the chills. Corticosteroids are not recommended because their immunosuppressive properties may suppress the antitumoricidal activity of mifamurtide. An algorithm to address these symptoms is proposed in Figure 1.

Finally, the role for mifamurtide in metastatic or recurrent osteosarcoma has not been fully evaluated. Future study of the use of mifamurtide in patients with newly diagnosed osteosarcoma and metastatic disease is warranted. Although such an endeavor will require a high level of participation from multiple institutions for this orphan indication, this may be the best means to provide a "confirmatory trial" to facilitate future approval of mifamurtide by the Food and Drug Administration.

\section{Disclosure}

The authors report no conflicts of interest in this work.

\section{References}

1. Mirabello L, Troisi RJ, Savage SA. Osteosarcoma incidence and survival rates from 1973 to 2004: data from the Surveillance, Epidemiology, and End Results Program. Cancer. 2009;115(7):1531-1543.

2. Anninga JK, Gelderblom H, Fiocco M, et al. Chemotherapeutic adjuvant treatment for osteosarcoma: where do we stand? Eur J Cancer. 2011; 47(16):2431-2445.

3. Bielack SS, Kempf-Bielack B, Delling G, et al. Prognostic factors in high-grade osteosarcoma of the extremities or trunk: an analysis of 1,702 patients treated on neoadjuvant cooperative osteosarcoma study group protocols. J Clin Oncol. 2002;20(3):776-790.

4. Daw NC, Neel MD, Rao BN, et al. Frontline treatment of localized osteosarcoma without methotrexate: results of the St Jude Children's Research Hospital OS99 trial. Cancer. 2011;117(12):2770-2778.

5. Whelan J, McTiernan A, Cooper N, et al. Incidence and survival of malignant bone sarcomas in England 1979-2007. Int JCancer. 2012;131(4): E508-E517.

6. Coley WB. II. Contribution to the knowledge of sarcoma. Ann Surg. 1891;14(3):199-220.

7. Jeys LM, Grimer RJ, Carter SR, Tillman RM, Abudu A. Post operative infection and increased survival in osteosarcoma patients: are they associated? Ann Surg Oncol. 2007;14(10):2887-2895.
8. Lee JA, Kim MS, Kim DH, et al. Postoperative infection and survival in osteosarcoma patients. Ann Surg Oncol. 2009;16(1):147-151.

9. Moore C, Eslin D, Levy A, Roberson J, Giusti V, Sutphin R. Prognostic significance of early lymphocyte recovery in pediatric osteosarcoma. Pediatr Blood Cancer. 2010;55(6):1096-1102.

10. De Angulo G, Hernandez M, Morales-Arias J, et al. Early lymphocyte recovery as a prognostic indicator for high-risk Ewing sarcoma. J Pediatr Hematol Oncol. 2007;29(1):48-52.

11. Arndt CA, Koshkina NV, Inwards CY, et al. Inhaled granulocytemacrophage colony stimulating factor for first pulmonary recurrence of osteosarcoma: effects on disease-free survival and immunomodulation. A report from the Children's Oncology Group. Clin Cancer Res. 2010; 16(15):4024-4030.

12. Muller CR, Smeland S, Bauer HC, Saeter G, Strander H. Interferonalpha as the only adjuvant treatment in high-grade osteosarcoma: long term results of the Karolinska Hospital series. Acta Oncol. 2005;44(5): 475-480.

13. Strander H, Bauer HC, Brosjo O, et al. Long-term adjuvant interferon treatment of human osteosarcoma. A pilot study. Acta Oncol. 1995; 34(6):877-880.

14. Winkler K, Beron G, Kotz R, et al. Neoadjuvant chemotherapy for osteogenic sarcoma: results of a Cooperative German/Austrian study. J Clin Oncol. 1984;2(6):617-624.

15. Asano T, Matsushima K, Kleinerman ES. Liposome-encapsulated muramyl tripeptide up-regulates monocyte chemotactic and activating factor gene expression in human monocytes at the transcriptional and posttranscriptional levels. Cancer Immunol Immunother. 1994;38(1):16-22.

16. Nardin A, Lefebvre ML, Labroquere K, Faure O, Abastado JP. Liposomal muramyl tripeptide phosphatidylethanolamine: targeting and activating macrophages for adjuvant treatment of osteosarcoma. Curr Cancer Drug Targets. 2006;6(2):123-133.

17. Fogler WE, Fidler IJ. Comparative interaction of free and liposomeencapsulated nor-muramyl dipeptide or muramyl tripeptide phosphatidylethanolamine (3H-labelled) with human blood monocytes. Int J Immunopharmacol. 1987;9(2):141-150.

18. Sone S, Mutsuura S, Ogawara M, Tsubura E. Potentiating effect of muramyl dipeptide and its lipophilic analog encapsulated in liposomes on tumor cell killing by human monocytes. J Immunol. 1984;132(4): 2105-2110.

19. Fidler IJ, Brown NO, Hart IR. Species variability for toxicity of free and liposome-encapsulated muramyl peptides administered intravenously. J Biol Response Mod. 1985;4(3):298-309.

20. Chi SN, Conklin LS, Qin J, et al. The patterns of relapse in osteosarcoma: the Memorial Sloan-Kettering experience. Pediatr Blood Cancer. 2004;42(1):46-51.

21. Crompton BD, Goldsby RE, Weinberg VK, Feren R, O’Donnell RJ, Ablin AR. Survival after recurrence of osteosarcoma: a 20-year experience at a single institution. Pediatr Blood Cancer. 2006;47(3): $255-259$.

22. Anderson P. Liposomal muramyl tripeptide phosphatidyl ethanolamine: ifosfamide-containing chemotherapy in osteosarcoma. Future Oncol. 2006;2(3):333-343.

23. Shiratsuchi A, Nakanishi Y. Phosphatidylserine-mediated phagocytosis of anticancer drug-treated cells by macrophages. J Biochem. 1999;126(6): 1101-1106.

24. Murray JL, Kleinerman ES, Cunningham JE, et al. Phase I trial of liposomal muramyl tripeptide phosphatidylethanolamine in cancer patients. J Clin Oncol. 1989;7(12):1915-1925.

25. Landmann R, Obrist R, Denz H, et al. Pharmacokinetics and immunomodulatory effects on monocytes during prolonged therapy with liposomal muramyltripeptide. Biotherapy. 1993;7(1):1-12.

26. Strober W, Murray PJ, Kitani A, Watanabe T. Signalling pathways and molecular interactions of NOD1 and NOD2. Nat Rev Immunol. 2006; 6(1):9-20.

27. Girardin SE, Boneca IG, Viala J, et al. Nod2 is a general sensor of peptidoglycan through muramyl dipeptide (MDP) detection. $J$ Biol Chem. 2003;278(11):8869-8872. 
28. Hedl M, Abraham C. Distinct roles for Nod2 protein and autocrine interleukin-1beta in muramyl dipeptide-induced mitogen-activated protein kinase activation and cytokine secretion in human macrophages. J Biol Chem. 2011;286(30):26440-26449.

29. Hu C, Sun L, Hu Y, Lu D, Wang H, Tang S. Functional characterization of the NF-kappaB binding site in the human NOD2 promoter. Cell Mol Immunol. 2010;7(4):288-295.

30. Kleinerman ES, Erickson KL, Schroit AJ, Fogler WE, Fidler IJ. Activation of tumoricidal properties in human blood monocytes by liposomes containing lipophilic muramyl tripeptide. Cancer Res. 1983; 43(5):2010-2014.

31. Dieter P, Ambs P, Fitzke E, Hidaka H, Hoffmann R, Schwende H. Comparative studies of cytotoxicity and the release of TNF-alpha, nitric oxide, and eicosanoids of liver macrophages treated with lipopolysaccharide and liposome-encapsulated MTP-PE. J Immunol. 1995;155(5):2595-2604.

32. Asano T, McWatters A, An T, Matsushima K, Kleinerman ES. Liposomal muramyl tripeptide up-regulates interleukin-1 alpha, interleukin-1 beta, tumor necrosis factor-alpha, interleukin-6 and interleukin-8 gene expression in human monocytes. J Pharmacol Exp Ther. 1994;268(2):1032-1039.

33. Dieter P, Hempel U, Kamionka S, et al. Prostaglandin E2 affects differently the release of inflammatory mediators from resident macrophages by LPS and muramyl tripeptides. Mediators Inflamm. 1999;8(6):295-303.

34. Maeda M, Knowles RD, Kleinerman ES. Muramyl tripeptide phosphatidylethanolamine encapsulated in liposomes stimulates monocyte production of tumor necrosis factor and interleukin-1 in vitro. Cancer Commun. 1991;3(10-11):313-321.

35. Asano T, McIntyre BW, Bednarczyk JL, Wygant JN, Kleinerman ES. Liposomal muramyl tripeptide upregulates adhesion molecules on the surface of human monocytes. Oncol Res. 1995;7(5):253-257.

36. Kleinerman ES, Raymond AK, Bucana CD, et al. Unique histological changes in lung metastases of osteosarcoma patients following therapy with liposomal muramyl tripeptide (CGP 19835A lipid). Cancer Immunol Immunother. 1992;34(4):211-220.

37. Asano T, Fujimaki W, McWatters A, An T, Matsushima K, Kleinerman ES. Effect of Adriamycin on liposomal muramyl tripeptide's ability to upregulate monocyte cytokine expression. Cancer Immunol Immunother. 1993;37(6):408-411.

38. Bezault J, Walsh C, Tarcsay L, Frost H, Liebes L, Furmanski P. Analysis of the antimetastatic effects of synthetic muramyl tripeptide (CGP 19835A) encapsulated in liposomes in combination with other immunomodulatory agents and chemotherapeutic drugs. In Vivo. 1993; 7(6A):487-491.

39. Killion JJ, Kleinerman ES, Wilson MR, Tanaka M, Fidler IJ. Sequential therapy with chemotherapeutic drugs and liposome-encapsulated muramyl tripeptide: determination of potential interactions between these agents. Oncol Res. 1992;4(10):413-418.

40. Kleinerman ES, Snyder JS, Jaffe N. Influence of chemotherapy administration on monocyte activation by liposomal muramyl tripeptide phosphatidylethanolamine in children with osteosarcoma. JClin Oncol. 1991;9(2):259-267.
41. Hisano G, Fidler IJ. Systemic activation of macrophages by liposome-entrapped muramyl tripeptide in mice pretreated with the chemotherapeutic agent adriamycin. Cancer Immunol Immunother. 1982;14(2):61-66.

42. Fujimaki W, Griffin JR, Kleinerman ES. Effect of ibuprofen on monocyte activation by liposome-encapsulated muramyl tripeptide phosphatidylethanolamine (CGP 19835A): can ibuprofen reduce fever and chills without compromising immune stimulation? Cancer Immunol Immunother. 1993;36(1):45-51.

43. Ando K, Mori K, Corradini N, Redini F, Heymann D. Mifamurtide for the treatment of nonmetastatic osteosarcoma. Expert Opin Pharmacother. 2011;12(2):285-292.

44. Meyers PA. Muramyl tripeptide (mifamurtide) for the treatment of osteosarcoma. Expert Rev Anticancer Ther. 2009;9(8):1035-1049.

45. Kleinerman ES, Meyers PA, Raymond AK, Gano JB, Jia SF, Jaffe N. Combination therapy with ifosfamide and liposome-encapsulated muramyl tripeptide: tolerability, toxicity, and immune stimulation. J Immunother Emphasis Tumor Immunol. 1995;17(3):181-193.

46. Creaven PJ, Cowens JW, Brenner DE, et al. Initial clinical trial of the macrophage activator muramyl tripeptide-phosphatidylethanolamine encapsulated in liposomes in patients with advanced cancer. $J$ Biol Response Mod. 1990;9(5):492-498.

47. Kleinerman ES, Jia SF, Griffin J, Seibel NL, Benjamin RS, Jaffe N. Phase II study of liposomal muramyl tripeptide in osteosarcoma: the cytokine cascade and monocyte activation following administration. J Clin Oncol. 1992;10(8):1310-1316.

48. Meyers PA, Schwartz CL, Krailo M, et al. Osteosarcoma: a randomized, prospective trial of the addition of ifosfamide and/or muramyl tripeptide to cisplatin, doxorubicin, and high-dose methotrexate. J Clin Oncol. 2005;23(9):2004-2011.

49. Urba WJ, Hartmann LC, Longo DL, et al. Phase I and immunomodulatory study of a muramyl peptide, muramyl tripeptide phosphatidylethanolamine. Cancer Res. 1990;50(10):2979-2986.

50. Meyers PA, Schwartz CL, Krailo MD, et al. Osteosarcoma: the addition of muramyl tripeptide to chemotherapy improves overall survival a report from the Children's Oncology Group. J Clin Oncol. 2008;26(4): 633-638.

51. Chou AJ, Kleinerman ES, Krailo MD, et al. Addition of muramyl tripeptide to chemotherapy for patients with newly diagnosed metastatic osteosarcoma: a report from the Children's Oncology Group. Cancer. 2009; $115(22): 5339-5348$

52. Anderson PM, Wiseman GA, Dispenzieri A, et al. High-dose samarium153 ethylene diamine tetramethylene phosphonate: low toxicity of skeletal irradiation in patients with osteosarcoma and bone metastases. J Clin Oncol. 2002;20(1):189-196.

53. Loeb DM, Garrett-Mayer E, Hobbs RF, et al. Dose-finding study of ${ }^{153} \mathrm{Sm}-\mathrm{EDTMP}$ in patients with poor-prognosis osteosarcoma. Cancer. 2009;115(11):2514-2522.
Clinical Oncology in Adolescents and Young Adults

\section{Publish your work in this journal}

Clinical Oncology in Adolescents and Young Adults is an international, peer-reviewed, open access journal publishing original research, reports, editorials, reviews and commentaries on all aspects of epidemiology, diagnosis and treatment of cancers in adolescents and young adults.

\section{Dovepress}

Visit http://www.dovepress.com/testimonials.php to read real quotes from published authors. 